\title{
On-line computers in psychology: A laboratory course for advanced psychology majors
}

\author{
DAVID M. SARGENT \\ State University of New York, Oswego, New York
}

\begin{abstract}
This course is structured as a series of increasingly difficult projects which involve students in all as pects of using computers for experimentation. Projects range from programming simple experiments utilizing monitor displays and keyboard responses to the use of hardware interfaces to connect complex peripherals. Students not only design and develop their projects, but also engage in data collection and analysis. The course provides experience with hardware and software as well as the intricacies of debugging the resulting project.
\end{abstract}

The primary purpose of the course On-Line Computers in Psychology is to familiarize the student with the methods used in computer-controlled psychological experimentation. It is designed to provide hands-on experience in all phases of psychological experimentation that require computer use. A basic understanding of computer architecture, real-time programming, computer interfacing, and an appreciation of how computers enhance psychological research are among the many benefits of this course.

Each student in the course is required to engage in all projects and to take part in all aspects of these projects. Project aspects usually fall into one of three categories: hardware, software, or experimental design. The individual projects in the beginning of the course provide the skills necessary for the more complex projects that follow. For example, in a first project, students program a routine to present a word in the center of the screen. The second project requires an array of words to be presented for specific durations on the screen. After a couple of other programming skills have been mastered, such as response inputting, the students are ready to design a program involving recall or recognition.

The two prerequisites for this upper division course are statistics and the methodology course required for the psychology major. Given the diversity of computing facilities on the campus, a specific prerequisite course involving computers was not feasible. Owing to the low enrollment capacity of 12 students, evaluation is based primarily on observed participation, with additional summative evaluations through experimental papers and presentations. Occasional practical tests such as wiring, soldering, programming, and debugging are also used for evaluation.

\section{Course History}

The On-Line Computers course was initially developed at the present institution in the late 1970s. At that time, the reigning operating system was $\mathrm{CP} / \mathrm{M}$ (Control Program for Microcomputers) for anyone wishing to connect the micro-

Correspondence should be addressed to D. M. Sargent, Department of Psychology, State University of New York, Oswego, NY 13126 (e-mail: sargent@oswego.oswego.edu). computer affordably to electromechanical equipment (Sargent, 1989). The $\mathrm{S}-100$ bus was available on select $\mathrm{CP} / \mathrm{M}$ machines such as NorthStar Horizon and Zenith, allowing limited access to relay operation and switch sensing. The ISA bus associated with 8086 and 8088 processors (primarily IBM and early IBM clones), while available, was cost prohibitive for college laboratories in psychology.

The initial laboratory configuration utilized six NorthStar Horizon CP/M computers. The NorthStar was chosen primarily for the ease of access to the $S-100$ bus, oversize power supply, compatibility with third-party peripherals, and overall cost. To facilitate graphical presentation of stimuli, Electrohome monochrome monitors driven by Paragraphics video adapter cards were used. The Paragraphics company also produced an analog/digital S-100 interface card, allowing electrical connection to external equipment.

Various transitional computers have been utilized in the course, including Radio Shack TRS-80s, KayPro CP/M machines, the Apple II and IIe, Commodore C64s, the Macintosh IIci (Newbus), and AT\&T 7300s (UNIX based) (Sargent, 1991). Many of these machines afforded interfacing to external equipment, but the biggest issue was overall compatibility. Over time, it became apparent that the ISA bus, MS-DOS-based machines were the most compatible, accessible, and best supported.

The course is conducted in a laboratory which currently has nine Gateway 80486 computers, running at $25-$ $33 \mathrm{MHz}$, each connected to an Alpha Products Interface Bus. Several companies currently produce low-cost interface devices that connect to the microcomputer through either a bus slot (ISA, VESA, and PCI) or the parallel or serial port. The interface allows computer control of any electrical switch or device, including the standard $28-\mathrm{Vdc}$ electromechanical equipment found in many psychology laboratories. The standard electromechanical equipment such as that produced by Gerbrands, Lehigh Valley Electronics (LVE), and Coulbourn, offers a highly flexible environment for the presentation of stimuli and the acquisition of subject responses through a wide variety of manipulanda. The barpress, lighted keypress, and panelpress (LVE) work equally well across subjects from humans to 
rats to pigeons. The variety of visual cues available (bulb clusters, 12 position projectors) or auditory cues (Sonalerts, tone generators) is usually adequate for the students' design needs. More importantly, electrical connections can be made and changed easily with the original snap-lead wires. It has been noted that making use of antiquated equipment to enhance teaching is looked upon quite favorably by department chairs and administrators alike.

\section{Current Course Specifics}

The course has been most successful when divided into modules. These modules involve specific skills that the students must master to complete the projects. The largest modules typically focus on the use of the programming language, most recently QuickBasic (MicroSoft) or Full Segment Forth (shareware). We have had success with either QuickBasic or Full Segment Forth used exclusively during a semester but have found it counterproductive to try to fit both languages into a semester. Other modules include electromechanicals, soldering, circuits, interface connection and testing, relays, feedback, human factors, experimental design, and piloting.

Typically the course begins by orienting the students to the computer basics. Initial modules are designed to establish a base of understanding of hardware and software through copying to and from fixed disks and floppies, connecting and setting up peripherals, writing batch files, and physically opening the computer case to identify components. As a supplement to the mechanical orientation, discussion modules also include operating systems, bus architecture, binary systems, Boolean logic, cycle speeds, and interrupts. As more modules are covered, including initial programming, the students are able to engage in the specific projects mentioned previously.

The programming language modules are constantly under revision, partially in response to repeated software updates. In addition, there is some debate over which language is becoming more standardized or which is better suited for the course. While there are obvious advantages to programming in $\mathrm{C}$, numerous revisions as students learn and as projects evolve make compilation and linking impractical. The BASIC family of programming languages has been used on and off successfully since the CP/M computers were used. The latest version of QuickBasic has the beneficial features of a full screen editor and a compilation option, allowing an executable program file that can be easily transported across computers. Another language that has successfully transcended computers and operating systems is FORTH. This programming language is essentially an interpreter with some distinctive differences, the most notable of which is that programs are developed strictly as a series of subroutines, requiring a slightly different approach with successive modules. The latest version of Full Segment Forth has many desirable features, including the fact that it is very cost effective.

Modules concerning circuits and basic electricity (ac/dc, current, voltages) are kept at a functional level and are tailored to the specific applications. The circuitry issues are centered on the electromechanical systems and the important differences in interfacing across voltages. While many of the electrical connections can be made with the versatile snap-leads, familiarizing the students with soldering techniques has been beneficial. Soldering skills are particularly helpful for debugging a system or when a unique piece of apparatus is required, such as a particular warning light or response lever.

Interspersed throughout the course are modules on various levels of debugging and testing. Specific troubleshooting techniques in circuit testing, relay testing, switch debouncing, program debugging, and pilot testing at several levels have resulted in greater understanding and better overall project quality. Limited success has been achieved in incorporating modules on the use of multimeters and oscilloscopes for testing stubborn computer problems. It is important to note that through these experiences, students very often become more comfortable with the technology.

The final project in the course allows the students, either individually or in small teams, to develop an experiment of their own design. Under the guidance of the instructor, students design, program, debug, and run subjects (usually other class members or friends) in their own experiment. Projects have included modifications of basic paradigms such as spatial memory, a mental rotation experiment similar to that of Shepard and Metzler (1971), and a series of memory tasks resembling those by Sternberg (1966). Following final project data collection, statistical analyses are run, and the results are written up and presented to the class, along with commentary on the specific development process. On several occasions, students have developed experimental programs that have been used in the methodology course to collect data and demonstrate psychological paradigms.

\section{Conclusions}

The present course has enjoyed a steadily increasing demand (growth in the waiting list) and has recently been offered in both the fall and spring semesters rather than just the spring. The course provides direct benefits by bringing together the skills and knowledge that students acquire in their required major courses. Its many indirect benefits to the students include computer prowess and practical skills that can be transferred across a wide range of applications.

\section{REFERENCES}

SARGENT, D. M. (1989). Use of on-line computers in experimental psychology: Hardware considerations. Behavior Research Methods, Instruments, \& Computers, 21, 163-165.

SARGENT, D. M. (1991). An economical system incorporating obsolete computers in an operant conditioning laboratory. Behavior Research Methods, Instruments, \& Computers, 23, 273-276.

ShEPARD, R. N., \& METZLER, J. (1971, February 19). Mental rotation of three-dimensional objects. Science, 171, 701-703.

STERNBERG, S. (1966, August 5). High-speed scanning in human memory. Science, 153, 652-654.

(Manuscript received November 17, 1995; accepted for publication January 5, 1996.) 